\title{
Diabetes mellitus-induced male reproductive impairment: The role of natural products: A review
}

\author{
Victor Udo Nna ${ }^{1,2}$, Ainul Bahiyah Abu Bakar ${ }^{1}$, Mahaneem Mohamed ${ }^{1 *}$ \\ ${ }^{1}$ Department of Physiology, School of Medical Sciences, Universiti Sains Malaysia, 16150 Kubang Kerian, Kelantan, Malaysia. \\ ${ }^{2}$ Department of Physiology, Faculty of Basic Medical Sciences, College of Medical Sciences, University of Calabar, P.M.B. 1115, Calabar, Cross River \\ State, Nigeria.
}

\begin{tabular}{|c|c|}
\hline ARTICLE INFO & ABSTRACT \\
\hline Article history: & \multirow{9}{*}{$\begin{array}{l}\text { Diabetes mellitus }(\mathrm{DM}) \text { is a metabolic disorder characterized by persistent hyperglycaemia, with multi-organ } \\
\text { complications. DM is known to trigger oxidative stress in virtually all tissues of the body including male } \\
\text { reproductive organs. Several experimental and clinical studies have reported varying degrees of male } \\
\text { reproductive impairment in DM. Disruption in the hypothalamic-pituitary-gonadal axis, abnormal testicular } \\
\text { energy metabolism, testicular and epididymal oxidative stress and oxidative stress-induced germ cell apoptosis } \\
\text { have been reported in DM. The use of natural products in the treatment of various diseases is gaining more } \\
\text { attention in experimental and clinical studies for the management and/or treatment of various diseases. In this } \\
\text { review, the effects of DM on male reproductive system at pre-testicular, testicular and post-testicular levels are } \\
\text { discussed. The role of various natural products including whole plant extracts and some pure compounds } \\
\text { isolated from plants, with their suggested mechanism of actions is also reviewed. }\end{array}$} \\
\hline Received on: 25/04/2017 & \\
\hline Accepted on: 08/06/2017 & \\
\hline Available online: 30/09/2017 & \\
\hline Key words: & \\
\hline Diabetes mellitus, male & \\
\hline reproductive system, natural & \\
\hline product, apoptosis, oxidative & \\
\hline stress. & \\
\hline
\end{tabular}

\section{INTRODUCTION}

Diabetes mellitus (DM) is a chronic metabolic disorder with a multi-faceted pathogenicity and multi-organ complications. The World Health Organization (WHO) has estimated that the prevalence of DM worldwide will increase to 300 million by 2030 (King, 1999). In 1998, $60 \%$ of the world diabetic population (140 million) was in Asia (King, 1999). Reports from a survey conducted in Malaysia in 1997 showed that over $8 \%$ of the adult population had DM (Zaini, 2000). In a more recent survey, the prevalence of DM among adults in Malaysia was $15.2 \%$ in 2011, and was increased to $17.5 \%$ in 2015 (National Health and Morbidity Survey, 2015).

\footnotetext{
* Corresponding Author

Mahaneem Mohamed, Department of Physiology, School of Medical Sciences, Universiti Sains Malaysia, 16150 Kubang Kerian, Kelantan, Malaysia.Email: mahaneem @ usm.my
}

Until recently, much attention is not given to the effects of DM on reproductive function, since most of the therapeutic interventions are targeted at eliminating the causes of DM and restoring normal health. Nevertheless, it is also important to monitor the effects of the disease on reproductive function. Several complications have been reported following DM, with the implication of oxidative stress (anti-oxidant/oxidant imbalance) as the major factor that is responsible for these complications ( $\mathrm{La}$ Vignera et al., 2008). This oxidative stress in the reproductive tissues forms the basis of reproductive impairments observed in individuals with DM (Mallidis et al., 2011). DM induces molecular alterations which negatively affect sperm quality and function as well as fertility (Mallidis et al., 2011). La Vignera et al., (2008) in a retrospective study reported the prevalence of subfertility to be $51 \%$ among patients with DM. Another study on infertility among DM patients reported a prevalence of $35.1 \%$ in men with type 2 DM (Bener et al., 2009). Age- dependent erectile dysfunction has also been reported in diabetic males (Young et al., 2004). 
Data from clinical and animal studies have suggested that DM affects male reproductive system at 3 levels namely pretesticular (Suresh et al., 2012), testicular (Ghosh et al., 2014) and post-testicular levels (Young et al., 2004).

To date, a lot of research has been done to study the role of different natural products in male reproductive functions (Mohamed et al., 2011; Mohamed et al., 2012; Mohamed et al., 2013). Some studies have used whole plant extracts, thus taking advantage of the abundance of different phytochemicals which have several intervention targets, while others have used specific compounds isolated from plants. Several natural products significantly reversed DM-induced reproductive impairment in rats (Ghosh et al., 2014; Khaki et al., 2014). In this review, we emphasize the effects of DM on reproductive function parameters as well as the effects of various natural products on DM-induced reproductive impairment with their possible mechanism(s) of actions.

\section{CLASSIFICATION OF DIABETES MELLITUS}

Diabetes mellitus has been grouped into two main types, namely type 1 DM (T1DM) and type 2 DM (T2DM). T1DM occurs as a result of defective beta cells in the islets of Langerhans. The beta cell abnormality can result from destruction by viral infections, diseases and exposure to various toxic chemicals (Cooke and Plotnick, 2008). Its classical symptoms are polyphagia, polydipsia, polyuria and weight loss (Cooke and Plotnick, 2008). On the other hand, T2DM is characterized by persistently high blood glucose due to the presence of insulin resistance and usually with relative insulin deficiency. Some of the risk factors for T2DM are genetic predisposition, obesity, hyperlipidemia and history of gestational diabetes. In most cases, the onset of T2DM occurs between the ages of 50-60 years. Following the increasing prevalence of obesity in recent years, there has been a steady increase in the number of younger individuals (some $<20$ years old) with T2DM (Kumar et al., 2005).

\section{EFFECT OF DIABETES MELLITUS ON MALE REPRODUCTIVE FUNCTION}

Several animal models of DM have been used in the study of male reproductive function. They include type 1 diabetic rats [streptozotocin (STZ)-induced diabetic rats, alloxan-induced diabetic rats and BioBreeding (BB) rats], type 2 diabetic rats [nicotinamide+STZ-induced diabetic rats (simultaneous administration of nicotinamide and STZ), STZ+high fats diet induced diabetic rats and Goto-Kakizaki (GK) rats]. DM affects male reproductive system at pre-testicular, testicular and posttesticular levels, which are summarized in Figure 1.

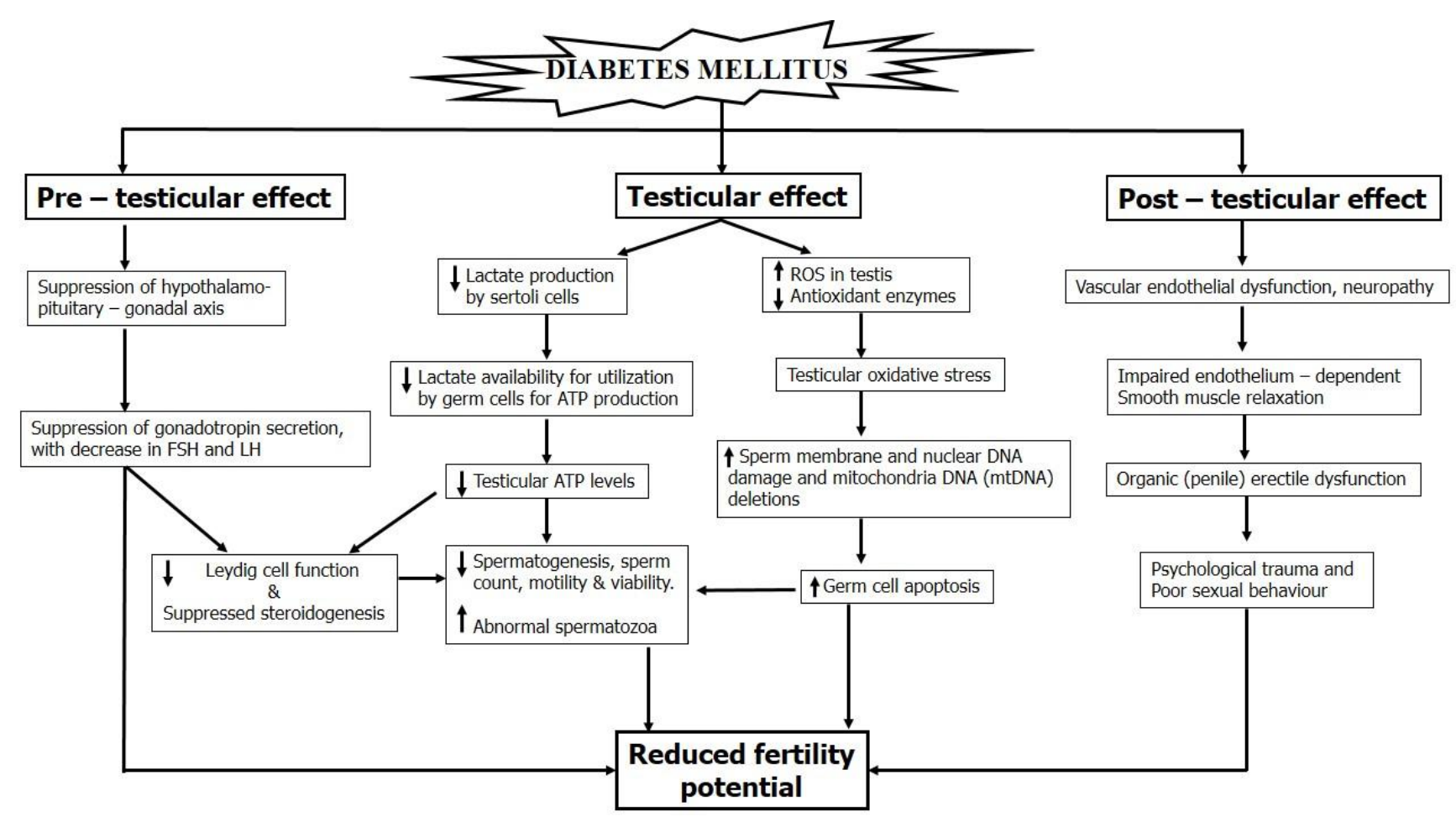

Fig. 1: Summary on the effect of DM on male reproductive system. 


\section{Pre-testicular effects of diabetes mellitus (hypothalamic- pituitary-gonadal axis)}

Several reports have linked DM with disruption of the hypothalamic-pituitary-gonadal (HPG) axis, thus altering the concentrations of testosterone, follicle stimulating hormone (FSH) and luteinizing hormone (LH) in males. Baccetti et al. (2002) have shown that DM significantly suppresses the HPG axis and lowers FSH and LH responses to exogenous gonadotropin releasing hormone $(\mathrm{GnRH})$ in T1DM patients. Suppressed testosterone secretion as a result of diminished Leydig cell function (Pitteloud et al., 2005) has been reported in T2DM. Decreased serum concentrations of reproductive hormones (testosterone, FSH and $\mathrm{LH})$ have also been reported in DM animal models induced by STZ (Mohasseb et al., 2011; Kanter et al., 2013), alloxan (Hafez, 2010; Ghlissi et al., 2012) and nicotinamide+STZ (Ahangarpour et al., 2015), and in BB rats (Cameron et al., 1990). These studies show a unified pattern of events irrespective of the diabetic model used.

The effect of DM on the HPG axis may not be type specific since the data from both $\mathrm{T} 1$ and $\mathrm{T} 2 \mathrm{DM}$ models have shown similar findings.

\section{Testicular effects}

\section{Energy metabolism in the testis}

The Sertoli cells (otherwise called nurse cells) are greatly involved in testicular energy metabolism, and form one of the major components of the blood - testis barrier. Testicular energy metabolism shows some form of specificity in which lactate is the main substrate for energy (ATP) production in germ cells (Oliveira et al., 2012). In vitro studies with cultured Sertoli cells have shown that removing either insulin (Oliveira et al., 2012) or glucose (Riera et al., 2009) from the culture medium results in adaptation of the Sertoli cells to glucose transport as seen in modulated gene expression of glucose transporters (GLUT1 and GLUT3). However, Oliveira et al., (2012) have reported that the insulindeprived Sertoli cells in their in vitro experiment (which closely mimics T1DM) have reduced glucose utilization even when the gene expression of the glucose transporters is increased. Additionally, the authors have reported down-regulation of the genes associated with lactate metabolism and transport. Recent study by Rato et al. (2015) has demonstrated that exposure of cultured Sertoli cells (obtained from T2 diabetic rats) to testosterone increases glucose consumption and up-regulates GLUT3, but down regulates GLUT1 gene expression compared with Sertoli cells culture obtained from pre-diabetic rats. There is also a decrease in protein expression of monocarboxylate transporter-4 (MCT4) and lactate dehydrogenase (LDH), as well as a decrease in LDH activity in Sertoli cell culture obtained from T2 diabetic rats, compared with Sertoli cell culture obtained from pre-diabetic rats (Rato et al., 2015).

In vivo studies using STZ-induced T1DM have reported that DM reduces testicular LDH activity (Kyathanahalli and Manjunath, 2014), thus suggesting a likely similar trend of events in T1 and T2DM. This ultimately implies that the germ cells may be deprived of lactate in diabetic conditions.

\section{Testicular oxidative stress}

The presence of antioxidants in the testis ensures that the two most important events in the testis namely steroidogenesis and spermatogenesis are not negatively affected by oxidative stress. These antioxidants in the testis are of major significance since oxidative stress is currently regarded as the most important cause of impaired testicular function underlying the pathological consequences of a wide range of conditions including DM. Studies with both $\mathrm{T} 1$ and $\mathrm{T} 2$ diabetic animal models have shown significant decreases in antioxidant enzymes and a significant increase in lipid peroxidation in the testis. Reports from studies using STZ-induced T1DM rats have shown significant decreases in testicular superoxide dismutase (SOD), catalase (CAT) and glutathione peroxidase (GPx) activities, and a significant increase in malondialdehyde (MDA) level (Mohasseb et al., 2011; Kanter et al., 2013).

In another study using alloxan-induced T1DM rats, Ghlissi et al. (2012) have reported significant decreases in the activities of antioxidant enzymes (SOD, CAT and GPx) and a significant increase in thiobarbituric acid-reactive substance level in the testis. A similar trend of results is reported by Hamden et al. (2008) using alloxan-induced T1DM rats. On the other hand, Gobbo et al. (2015) have reported that DM did not significantly alter the testicular antioxidant defence system as shown by the insignificant alterations in the activities of antioxidant enzymes (SOD, CAT, GPx) and MDA level after 1 week and 8 weeks in STZ-induced T1DM rats.

\section{Steroidogenesis}

Steroid hormones including testosterone are synthesized from cholesterol through a series of tightly regulated steps. After formation of testosterone, the Leydig cells are involved with its secretion to support spermatogenesis. Studies have reported a significantly increased testicular cholesterol concentration in STZinduced T1DM model (Saumya and Basha, 2016). Significant decreases in $3 \beta$-hydroxysteroid dehydrogenase (HSD) and $17 \beta$ HSD activities are found in the testis of STZ-induced T1DM rat model (Reddy et al., 2016). Furthermore, there are significant decreases in expressions of steroidogenic acute regulatory protein (StAR) and cytochrome P450 enzyme (CYP11A1, the first enzymatic step in steroidogenesis) in the testis of STZ-induced T1DM rats.

The increased testicular cholesterol concentration (Saumya and Basha, 2016) may be as a result of down-regulation of the cholesterol transporter, StAR, thus leading to cholesterol accumulation. These results suggest that the reduced serum testosterone concentration in DM amidst high testicular cholesterol concentration may be due to down-regulations of steroidogenic transport protein (StAR) and marker enzymes (CYP11A1, 3 $\beta$-HSD and $17 \beta-\mathrm{HSD}$ ) expressions. 


\section{Effect of diabetes mellitus on spermatogenesis}

Experimental (Sangameswaran and Jayakar, 2008; Reddy et al., 2016) and clinical (Delfino et al., 2007) evidences have shown that DM negatively affects spermatogenesis and sperm related parameters. Several studies using STZ-induced T1DM animal model have shown decreased daily sperm production, sperm count and motility (Suresh and Prakash, 2012; Gonzales et al., 2013), and increased percentage of spermatozoa with abnormal morphology (Kanter et al., 2013). Studies using alloxan-induced T1DM animal models (Hafez 2010; Ghlissi et al., 2012) have also yielded similar results.

Reports from clinical studies have failed to completely corroborate those from experimental animals, and most of them have yielded ambiguous results. Vignon et al. (1990) have previous reported a significant increase in sperm concentration in TIDM patients, while Garcia-Diez (1991) has reported a significant decrease in sperm count, motility and percentage of spermatozoa with normal morphology. However, Agbaje et al. (2007) have found an insignificant increase in sperm concentration and total sperm output with an insignificant decrease in sperm motility, and normal sperm morphology. The authors suggested Leydig cell hyperplasia as the cause of the enhanced spermatogenesis, though with poor motility. These inconsistent findings might be as a result of the lack of information with regards to the duration of exposure to DM and the various control measures adopted by the subjects in each population studied, since most of these studies were conducted using patients reporting to fertility clinics, rather than untreated diabetic volunteers.

\section{Testicular histology and germ cell apoptosis in diabetes mellitus}

Oxidative stress, which is one of the hallmarks of DM, has been implicated in DM - induced distorted testicular morphology in diabetic animal models. Studies using T1 (Kanter et al., 2013; Al-Roujeaie et al., 2016) and T2 (Long et al., 2015) diabetic rats have yielded similar results, indicating that testicular histology is negatively affected irrespective of the type of DM. Studies using STZ (Kanter et al., 2013; Al-Roujeaie et al., 2016), alloxan (Shalaby and Hamowieh, 2010; Hafez, 2010) and BB (Cameron et al., 1990) T1DM models have all reported varying degrees of morphological abnormalities, comparable to the study using T2DM (STZ + High fats diet) model (Long et al., 2015). Mean Johnsen's score, a measure of the degree of spermatogenesis, is significantly lower in STZ- induced T1DM model (Kanter et al., 2012; 2013). Testicular atrophy has also been reported in DM with significant decreased volume and diameter of the seminiferous tubule, and decreased numbers of Sertoli and Leydig cells (Kanter et al., 2013; Al-Roujeaie et al., 2016). Kanter et al. (2013) have also reported a significantly decreased population of germ cells including spermatogonia, spermatocytes and spermatids at various stages which may explain the decreased testicular weight in DM.

The increased population of apoptotic germ cells observed in diabetic rats is suggestive of the implication of oxidative stress that usually accompanies DM. Mohasseb et al. (2011) have reported an increased activity of the executioner caspase (caspase-3) in the testis of STZ-induced diabetic rats. Upregulation of the pro-apoptotic protein (Bax) and down-regulation of the anti-apoptotic protein (Bcl-2) have been reported in $\mathrm{T} 1$ (Ghosh et al., 2014) and T2 (Long et al., 2015) diabetic rats, with an increase in expression of caspases 8 and 3 (Jiang et al., 2013) in T1 diabetic rats. Zhao et al. (2011) have reported up-regulation of the pro-apoptotic genes, p38 and p53 in the testis of STZ-induced T1DM rats. Studies in detecting apoptotic germ cells in the testis of T1 diabetic rats using TUNEL staining technique have reported an increased expression of TUNEL-positive cells (Kanter et al., 2012; 2013).

In the light of the above results, it is clear that all the methods and/or markers used to assess DM- induced germ cell apoptosis show uniformity in the trend of results obtained, thus confirming the increased apoptotic germ cell population in diabetic state.

\section{Post-testicular effects of diabetes mellitus (Sexual behaviour and fertility outcome)}

Studies have linked DM with poor sexual urge as a consequence of poor penile erectile function. Studies have shown that DM reduces penile cGMP concentration, prolongs mount, intromission and ejaculatory latencies, and decreases mount and intromission frequencies (Al-Roujeaie et al., 2016). The decreases in sexual behavioural parameters are linked to the reduced Leydig cell secretion of testosterone (Al-Roujeaie et al., 2016). In another study, diabetic male rats have demonstrated subfertility when mated with healthy female rats (Suresh and Prakash, 2012; Reddy et al., 2016).

Reddy et al. (2016) have reported an increase in the percentage of pre- and post-implantation losses, and a decrease in the number of live foetuses/dam. DM does not negatively affect pregnancy rate, but prolongs conception time, which is attributed to poor sperm characteristics secondary to DM (Reddy et al., 2016). Taken together, these reports implicate abnormal sexual behaviour in DM- mediated subfertility.

\section{NATURAL PRODUCTS AND DM-INDUCED IMPAIRMENT IN MALE REPRODUCTIVE FUNCTION}

Natural products and phytotherapy have been widely embraced lately, probably because of the perceived effectiveness relative to modern medications. This is possibly because of the abundance of various phytochemicals, which have multiple intervention targets in the course of treatment of the targeted condition. Several natural products have been employed in the treatment or management of DM and its accompanying complications. In the subsections below, we also review the role of various natural products [whole extracts (Table 1) and pure compounds isolated from plants (Table 2)] in ameliorating DMinduced male reproductive abnormalities. 
Table 1: Selected studies reporting the effect of natural products on DM - induced reproductive impairment.

\begin{tabular}{|c|c|c|c|c|c|c|}
\hline Diabetic Model & $\begin{array}{l}\text { Type of } \\
\text { DM }\end{array}$ & $\begin{array}{l}\text { Natural product } \\
\text { (part used) }\end{array}$ & $\begin{array}{l}\text { Dose } \\
\text { (duration of } \\
\text { treatment) }\end{array}$ & $\begin{array}{l}\text { Anti-diabetic } \\
\text { Standard }\end{array}$ & $\begin{array}{l}\text { Effect on } \\
\text { FBG }\end{array}$ & $\begin{array}{l}\text { Outcome on Reproductive function } \\
\text { parameters }\end{array}$ \\
\hline \multirow[t]{22}{*}{$\begin{array}{l}\text { STZ-induced } \\
\text { DM }\end{array}$} & T1DM & $\begin{array}{l}\text { Amaranthus spinosus } \\
\text { (stem) }\end{array}$ & $\begin{array}{l}250 \text { and } \\
500 \mathrm{mg} / \mathrm{kg} / \text { day, } \\
(15 \text { days })\end{array}$ & Glibenclamide & $\downarrow \#$ & $\begin{array}{l}\uparrow \text { Testicular weight, testosterone, } \\
\text { spermatogenesis (Sangameswaran and } \\
\text { Jayakar 2008). }\end{array}$ \\
\hline & & Brazilian propolis & $\begin{array}{l}100 \mathrm{mg} / \mathrm{kg} / \mathrm{day}, \\
(8 \text { weeks })\end{array}$ & Glucobay & $\downarrow \#$ & Not investigated (Zhu et al., 2011). \\
\hline & & Chinese propolis & $\begin{array}{l}100 \mathrm{mg} / \mathrm{kg} / \mathrm{day} \\
(8 \text { weeks })\end{array}$ & Glucobay & $\downarrow \#$ & Not investigated (Zhu et al., 2011). \\
\hline & & $\begin{array}{l}\text { Chlorophytum } \\
\text { borivilianum (root) }\end{array}$ & $\begin{array}{l}200 \mathrm{mg} / \mathrm{kg} / \text { day, } \\
(28 \text { days })\end{array}$ & Glibenclamide & $\downarrow \#$ & $\begin{array}{l}\uparrow \text { Sperm count, motility, viability and \% } \\
\text { normal morphology; } \downarrow \text { oxidative stress and } \\
\text { apoptosis in sperm (Thakur et al., } 2009 \text { ). }\end{array}$ \\
\hline & & $\begin{array}{l}\text { Chlorophytum } \\
\text { borivilianum (root) }\end{array}$ & $\begin{array}{l}200 \mathrm{mg} / \mathrm{kg} / \mathrm{day} \\
\text { (28 days) }\end{array}$ & - & $\downarrow \#$ & $\uparrow$ Sexual behaviour (Thakur et al., 2009). \\
\hline & & $\begin{array}{l}\text { Chlorophytum } \\
\text { borivilianum (tuber) }\end{array}$ & $\begin{array}{l}100 \text { and } \\
300 \mathrm{mg} / \mathrm{kg} / \text { day, } \\
(14 \text { days })\end{array}$ & - & - & $\uparrow$ Sexual behaviour (Vyawahare et al., 2009). \\
\hline & & Cinnamon zeylanicum & $\begin{array}{l}75 \mathrm{mg} / \mathrm{kg} / \mathrm{day} \\
(8 \text { weeks) }\end{array}$ & - & $\downarrow$ & $\begin{array}{l}\uparrow \text { Serum testosterone, sperm count, motility } \\
\text { and viability (Khaki et al., 2014). }\end{array}$ \\
\hline & & Curculigo orchioides & $\begin{array}{l}100 \text { and } \\
200 \mathrm{mg} / \mathrm{kg} / \text { day } \\
(28 \text { days })\end{array}$ & Glibenclamide & $\downarrow \#$ & $\begin{array}{l}\uparrow \text { Serum testosterone, sperm count; improved } \\
\text { sexual behaviour and penile erection index } \\
\text { (Thakur } \text { et al., 2012). }\end{array}$ \\
\hline & & $\begin{array}{l}\text { Danae racemosa } \\
\text { (leaves) }\end{array}$ & $\begin{array}{l}400 \mathrm{mg} / \mathrm{kg} / \mathrm{day} \\
\text { (28 days) }\end{array}$ & - & - & $\begin{array}{l}\uparrow \text { Serum testosterone, testis weight (Shahreari } \\
\text { et al., 2010). }\end{array}$ \\
\hline & & $\begin{array}{l}\text { Dracaena arborea (root } \\
\text { bark) }\end{array}$ & $\begin{array}{l}100 \text { and } \\
500 \mathrm{mg} / \mathrm{kg} / \mathrm{day}, \\
(4 \text { weeks })\end{array}$ & - & $\downarrow$ & $\begin{array}{l}\uparrow \text { Sexual behaviour (Wankeu-Nya et al., } \\
\text { 2014). }\end{array}$ \\
\hline & & $\begin{array}{l}\text { Dracaena arborea (root } \\
\text { bark) }\end{array}$ & $\begin{array}{l}100 \text { and } \\
500 \mathrm{mg} / \mathrm{kg} / \text { day, } \\
(3 \text { weeks) }\end{array}$ & - & $\downarrow$ & $\begin{array}{l}\text { Improved erectile function (Wankeu-Nya et } \\
\text { al., 2013). }\end{array}$ \\
\hline & & $\begin{array}{l}\text { Eugenia jambolana } \\
\text { (seeds) }\end{array}$ & $\begin{array}{l}200 \mathrm{mg} / \mathrm{kg}, \text { twice daily, } \\
\text { (60 days) }\end{array}$ & - & - & $\begin{array}{l}\uparrow \text { Serum testosterone, testis weight and sperm } \\
\text { count; } \downarrow \text { Bax and } \uparrow \text { Bcl- } 2 \text { expression in the } \\
\text { testis; } \uparrow \text { germ cell regeneration (Ghosh } \text { et al., } \\
\text { 2014). }\end{array}$ \\
\hline & & $\begin{array}{l}\text { Hibiscus sabdariffa } \\
\text { (calyx) }\end{array}$ & $\begin{array}{l}100 \mathrm{mg} / \mathrm{kg} / \mathrm{day} \\
(28 \text { days })\end{array}$ & - & $\downarrow \#$ & $\begin{array}{l}\uparrow \text { Testicular weight, sperm count, motility; } \downarrow \\
\text { abnormal morphology (Idris et al., 2012). }\end{array}$ \\
\hline & & $\begin{array}{l}\text { Ligustrum lucidum } \\
\text { (fruit) }\end{array}$ & $\begin{array}{l}30 \mathrm{~g} / \mathrm{kg} / \mathrm{day}, \\
\text { (110 days) }\end{array}$ & - & - & $\begin{array}{l}\uparrow \text { Serum testosterone, FSH and LH (Feng et } \\
\text { al., 2001). }\end{array}$ \\
\hline & & $\begin{array}{l}\text { Mucuna pruriens } \\
\text { (seeds) }\end{array}$ & $\begin{array}{l}200 \mathrm{mg} / \mathrm{kg} / \mathrm{day} \\
\text { (60 days) }\end{array}$ & - & $\downarrow \#$ & $\begin{array}{l}\uparrow \text { Serum testosterone, FSH, LH; } \uparrow \text { weight of } \\
\text { testis, epididymis, prostrate and seminal } \\
\text { vesicle; } \uparrow \text { DSP, sperm count, motility and } \\
\text { viability; } \uparrow \text { erectile function and sexual } \\
\text { behaviour (Suresh and Prakash } 2012 \text { ). }\end{array}$ \\
\hline & & Musa paradisiaca (root) & $\begin{array}{l}200 \mathrm{mg} / \mathrm{kg} \text { twice daily, } \\
\text { (28 days) }\end{array}$ & Glibenclamide & $\downarrow \#$ & $\begin{array}{l}\uparrow \text { Serum testosterone; } \downarrow \text { testicular cholesterol; } \\
\uparrow \text { sperm count, motility; improved testicular, } \\
\text { epididymal and sperm antioxidant status; } \\
\text { decreased testicular germ cell apoptosis; } \\
\text { improved testicular histology (Chatterjee et } \\
\text { al., 2012). }\end{array}$ \\
\hline & & $\begin{array}{l}\text { Musa paradisiaca (root) } \\
+ \\
\text { Coccinia indica (leaves) }\end{array}$ & $\begin{array}{l}20 \mathrm{mg} / \mathrm{kg} / \mathrm{day}, \\
\text { (45 days) }\end{array}$ & - & $\downarrow \#$ & $\begin{array}{l}\uparrow \text { Serum testosterone; } \uparrow \text { sperm count and } \\
\text { viability; } \downarrow \text { testicular oxidative stress and } \\
\text { germ cell apoptosis (Mallick et al., 2010). }\end{array}$ \\
\hline & & $\begin{array}{l}\text { Parkia biglobosa } \\
\text { (seeds) }\end{array}$ & $\begin{array}{l}200 \text { and } \\
400 \mathrm{mg} / \mathrm{kg} / \mathrm{day},(28 \\
\text { days })\end{array}$ & Insulin & - & $\begin{array}{l}\uparrow \text { Serum testosterone; improved testicular } \\
\text { antioxidant (Ogunyinka et al., 2016). }\end{array}$ \\
\hline & & Sesame oil & $\begin{array}{l}5 \text { and } 10 \% \text { diet } \\
\text { (56 days) }\end{array}$ & - & $\uparrow$ & $\begin{array}{l}\uparrow \text { Serum testosterone, sperm motility and } \\
\text { viability; } \downarrow \text { abnormal morphology (Abbasi } \text { et } \\
\text { al., 2013). }\end{array}$ \\
\hline & & $\begin{array}{l}\text { Spirulina maxima } \\
\text { (pellets) }\end{array}$ & $\begin{array}{l}200 \mathrm{mg} / \mathrm{kg} / \mathrm{day}, \\
\text { (4 weeks) }\end{array}$ & - & $\downarrow$ & $\begin{array}{l}\uparrow \text { Testis weight, testosterone, Leydig cell } \\
\text { density, steroidogenesis (Nah } \text { et al., 2012). }\end{array}$ \\
\hline & & $\begin{array}{l}\text { Withania somnifera } \\
\text { (roots) }\end{array}$ & $\begin{array}{l}500 \mathrm{mg} / \mathrm{kg} / \mathrm{day} \\
(15 \text { days })\end{array}$ & - & $\downarrow \#$ & $\begin{array}{l}\uparrow \text { Testis weight, antioxidant enzymes and } \downarrow \\
\text { lipid peroxidation; } \uparrow \text { testicular LDH and } 3 \beta \text { - } \\
\text { HSD (Kyathanahalli and Manjunath 2014). }\end{array}$ \\
\hline & & $\begin{array}{l}\text { Zingiber officinale } \\
\text { (root) }\end{array}$ & $\begin{array}{l}100 \mathrm{mg} / \mathrm{kg} / \mathrm{day} \\
(8 \text { weeks) }\end{array}$ & - & $\downarrow$ & $\begin{array}{l}\uparrow \text { Serum testosterone, sperm count, motility } \\
\text { and viability (Khaki et al., 2014). }\end{array}$ \\
\hline
\end{tabular}




\begin{tabular}{|c|c|c|c|c|c|c|}
\hline $\begin{array}{l}\text { Alloxan - } \\
\text { induced } \\
\text { DM }\end{array}$ & T1DM & $\begin{array}{l}\text { Chlorophytum } \\
\text { borivilianum (root) }\end{array}$ & $\begin{array}{c}100 \text { and } \\
300 \mathrm{mg} / \mathrm{kg} / \text { day, } \\
\text { (28 days })\end{array}$ & - & $\downarrow \#$ & $\uparrow$ Sexual behaviour (Vyawahare et al., 2009). \\
\hline & & Nigella sativa (seeds) & $\begin{array}{l}2 \% \text { in diet } \\
\text { (30 days) }\end{array}$ & - & $\downarrow \#$ & $\begin{array}{l}\uparrow \text { Weight of testis, epididymis, seminal vesicle } \\
\text { and prostrate; } \uparrow \text { serum testosterone, sperm } \\
\text { count and motility; } \downarrow \text { testicular LDH and } \\
\text { oxidative stress (Ghlissi } \text { et al., 2012). }\end{array}$ \\
\hline & & $\begin{array}{l}\text { Zingiber officinale } \\
\text { (root) }\end{array}$ & $\begin{array}{c}100 \text { and } \\
200 \mathrm{mg} / \mathrm{kg} / \mathrm{day}, \\
(65 \text { days })\end{array}$ & - & $\downarrow$ & $\begin{array}{l}\uparrow \text { Serum testosterone, testis weight, sperm } \\
\text { count, sperm motility; } \downarrow \text { testicular lesions, } \uparrow \\
\text { fertility index (Shalaby and Hamowieh } 2010 \text { ). }\end{array}$ \\
\hline & & $\begin{array}{l}\text { Zingiber officinale } \\
\text { (root) }\end{array}$ & $\begin{array}{c}250 \text { and } \\
500 \mathrm{mg} / \mathrm{kg} / \mathrm{day} \\
(65 \text { days })\end{array}$ & - & $\downarrow$ & $\begin{array}{l}\uparrow \text { Weight of testis, seminal vesicle and } \\
\text { prostrate; } \uparrow \text { sperm count, motility, viability } \\
\text { and } \downarrow \text { abnormality (Hafez } 2010 \text { ). }\end{array}$ \\
\hline \multirow{2}{*}{$\begin{array}{l}\text { Nicotinam } \\
\text { ide }+ \text { STZ } \\
\text { - induced } \\
\text { DM }\end{array}$} & T2DM & Arctium lappa (root) & $\begin{array}{l}200 \text { and } \\
300 \mathrm{mg} / \mathrm{kg} / \mathrm{day} \\
\text { (28 days) }\end{array}$ & Glibenclamide & - & $\uparrow$ Sperm viability (Ahangarpour et al., 2015). \\
\hline & & Rhus coriaria (seeds) & $\begin{array}{c}200 \text { and } \\
400 \mathrm{mg} / \mathrm{kg} / \mathrm{day} \\
(28 \text { days })\end{array}$ & Glibenclamide & - & $\begin{array}{l}\uparrow \text { Serum testosterone, FSH, LH, testis weight, } \\
\text { sperm count, viability (Ahangarpour } \text { et al., } \\
2014 \text { ). }\end{array}$ \\
\hline
\end{tabular}

All treatments are given orally; \# represents a significant decrease in fasting blood glucose (FBG) level compared with the diabetic control group.

Table 2: Selected studies reporting effects of pure compounds isolated from plants on DM-induced reproductive impairment.

\begin{tabular}{|c|c|c|c|c|c|c|}
\hline $\begin{array}{l}\text { Diabetic } \\
\text { Model }\end{array}$ & $\begin{array}{l}\text { Type of } \\
\text { DM }\end{array}$ & $\begin{array}{c}\text { Compound } \\
\text { (class of compound) }\end{array}$ & $\begin{array}{c}\text { Dose } \\
\text { (duration } \\
\text { of treatment) }\end{array}$ & $\begin{array}{l}\text { Anti-diabetic } \\
\text { Standard }\end{array}$ & $\begin{array}{c}\text { Effect } \\
\text { on } \\
\text { FBG }\end{array}$ & $\begin{array}{l}\text { Outcome on Reproductive function } \\
\text { parameters }\end{array}$ \\
\hline \multirow[t]{7}{*}{$\begin{array}{l}\text { STZ-induced } \\
\text { DM }\end{array}$} & T1DM & Curcumin (phenol) & $\begin{array}{l}100 \mathrm{mg} / \mathrm{kg} / \mathrm{day} \\
\text { (8 weeks) }\end{array}$ & - & $\downarrow$ & $\begin{array}{l}\uparrow \text { Testicular weight, serum testosterone; } \downarrow \\
\text { testicular oxidative stress; improved testicular } \\
\text { cytoarchitecture and } \downarrow \text { germ cell apoptosis } \\
\text { (Kanter } \text { et al., 2013). }\end{array}$ \\
\hline & & $\begin{array}{l}\text { Naringenin } \\
\text { (flavanone) }\end{array}$ & $\begin{array}{l}5 \text { and } 10 \mathrm{mg} / \mathrm{kg} / \mathrm{day}, \\
(10 \text { weeks })\end{array}$ & - & $\downarrow \#$ & $\begin{array}{l}\uparrow \text { Serum testosterone, sperm count, motility, } \\
\text { viability; improve spermatogenesis; increase } \\
\text { Sertoli cell number; } \downarrow \text { testicular oxidative stress; } \\
\downarrow \text { testicular inflammation and apoptosis (Roy et } \\
\text { al., 2013). }\end{array}$ \\
\hline & & Quercetin (flavonoid) & $\begin{array}{l}15 \mathrm{mg} / \mathrm{kg} / \mathrm{day}, \text { ip } \\
\text { (4 weeks) }\end{array}$ & - & - & $\begin{array}{l}\uparrow \text { Sperm count, motility and viability; } \downarrow \text { sperm } \\
\text { oxidative stress (Khaki et al., 2009). }\end{array}$ \\
\hline & & Quercetin (flavonoid) & $\begin{array}{l}15 \mathrm{mg} / \mathrm{kg} / \mathrm{day}, \text { ip } \\
\text { (4 weeks) }\end{array}$ & - & $\downarrow$ & $\begin{array}{l}\uparrow \text { Testicular weight, serum testosterone, sperm } \\
\text { count, motility and viability; improved testicular } \\
\text { cytoarchitecture (Khaki et al., 2010). }\end{array}$ \\
\hline & & Quercetin (flavonoid) & $\begin{array}{l}15 \mathrm{mg} / \mathrm{kg} / \mathrm{day}, \text { ip } \\
\text { (8 weeks) }\end{array}$ & - & - & $\begin{array}{l}\uparrow \text { Testicular weight, serum testosterone; } \downarrow \\
\text { testicular glucose and oxidative stress, improved } \\
\text { testicular cytoarchitecture and } \downarrow \text { germ cell } \\
\text { apoptosis (Kanter et al., 2012). }\end{array}$ \\
\hline & & Resveratrol (phenol) & $\begin{array}{l}5 \mathrm{mg} / \mathrm{kg} / \mathrm{day}, \\
\text { (8 weeks) }\end{array}$ & - & $\bar{\uparrow}$ & $\begin{array}{l}\downarrow \text { Oxidative stress in corpus cavernosum, } \downarrow \\
\text { corporal tissue apoptosis and } \uparrow \text { penile erectile } \\
\text { function (Yu et al., 2013). }\end{array}$ \\
\hline & & Rutin (flavonoid) & $\begin{array}{c}50 \text { and } \\
100 \mathrm{mg} / \mathrm{kg} / \mathrm{day}, \\
(5 \text { weeks })\end{array}$ & - & $\downarrow$ & $\begin{array}{l}\uparrow \text { Testicular weight and serum testosterone; } \downarrow \\
\text { testicular oxidative stress; improved sexual } \\
\text { behaviour; improved testicular cytoarchitecture } \\
\text { (Al-Roujeaie et al., 2016). }\end{array}$ \\
\hline $\begin{array}{l}\text { STZ }+ \text { High } \\
\text { fats diet }- \\
\text { induced DM }\end{array}$ & T2DM & Scutellarin (phenol) & $\begin{array}{l}100 \mathrm{mg} / \mathrm{kg} / \mathrm{day}, \\
\text { (60 days) }\end{array}$ & - & $\downarrow$ & $\begin{array}{l}\uparrow \text { Testicular and epididymal weights, } \uparrow \text { testicular } \\
\text { microcirculation, } \downarrow \text { testicular oxidative stress, } \\
\text { improved testicular cytoarchitecture and } \downarrow \text { germ } \\
\text { cell apoptosis (Long } \text { et al., } 2015 \text { ). }\end{array}$ \\
\hline
\end{tabular}

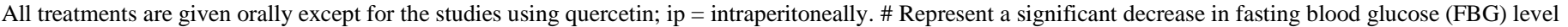
compared with the diabetic control group.

\section{Natural products with pre-testicular (HPG-axis) beneficial effects in diabetes mellitus}

Investigations using $\mathrm{T} 1$ diabetic rats have shown that administration of fruit extract of Ligustrum lucidum (Feng et al., 2001) or seed extract of Mucuna pruriens (Suresh and Prakash, 2012) for 60 days significantly increases serum concentrations of testosterone, FSH and LH. Seed extract of Rhus coriaria has been demonstrated to have a similar effect in T2 diabetic rats (Ahangarpour et al., 2015). All other plant extracts have been reported to have an effect on the HPG-axis but only limited their findings to testosterone concentration (Table 1). Studies using pure compounds of plant origin (Table 2) have also been reported to improve testosterone concentration in $\mathrm{T} 1$ and $\mathrm{T} 2$ diabetic animal models. Curcumin, a phenol, has been shown to increase serum 
testosterone concentration in $\mathrm{T} 1$ diabetic rats after 8 weeks of treatment (Kanter et al., 2013). Flavonoids like quercetin (Khaki et al., 2010; Kanter et al., 2012) and rutin (Al-Roujeaie et al., 2016) are reported to also improve serum testosterone concentration in T1 diabetic rats. Similarly, flavanone naringenin is shown to increase serum testosterone concentration in $\mathrm{T} 1$ diabetic rats after 10 weeks of administration (Roy et al., 2013). It is suggested that an increase in testosterone secretion helps in normal spermatogenesis for the production of normal and healthy spermatozoa.

\section{Natural products with testicular effects in diabetes mellitus}

The effects of DM on the testis seem to revolve around abnormal glucose metabolism in the testis and reduced availability of ATP for the two key events (steroidogenesis and spermatogenesis) in the testis. However, most of the studies on natural products do not show sufficient data on testicular energy metabolism in diabetic animal models. Only a few studies have reported their effects on LDH. Arikawe et al. (2012) have reported that administration of Zingiber officinale extract to STZ- induced T1 diabetic rats reverses the increased LDH activity in the testis. Kyathanahalli and Manjunath (2014) have also reported a similar finding after 15 days administration of the root extract of Withania somnifera. In a study using flavonoid quercetin, Kanter et al. (2012) have reported a reduced concentration of glucose in the testis homogenate of STZ - induced T1 diabetic rats after quercetin supplementation. This suggests that quercetin controls testicular hyperglycaemia. However, testicular glucose is the only testicular energy metabolism - related parameter assessed in this study thus making it difficult to suggest the mechanism of action. Some authors have suggested that an increase in tissue LDH activity is characteristic of tissue damage associated with exposure to xenobiotics (Srivastava and Pant, 2003). Therefore, assessment of LDH activity alone is not sufficient to demonstrate the effects of natural products on testicular glucose regulation.

A study on T1DM using Musa paradisiaca root extract has demonstrated decreased cholesterol level and increased testosterone in the testis of diabetic rats after 28 days of administration (Chatterjee et al., 2012). Increased expressions of StAR and $17 \beta-H S D$, with no significant effect on $3 \beta-H S D$, in addition to increased serum testosterone concentration are found in T1 diabetic rats treated with Spirulina maxima for 4 weeks. It is suggested that with the increased StAR protein expression compared to the DM group, more cholesterol would be delivered to the mitochondria of Leydig cells for testosterone synthesis (Nah et al., 2012). Kyathanahalli and Manjunath (2014) have reported an increase in expression of $3 \beta$-HSD in T1 diabetic rats following 15 days administration of Withania somnifera. Nevertheless, studies using other plant extracts have no reports showing their effects on the markers of steroidogenesis, even though some have reported an improved serum concentration of testosterone (Table 1). Spermatogenesis is negatively affected when steroidogenesis is suppressed, as observed in DM. Studies using whole plant extracts
(Mallick et al., 2010; Hafez 2010; Gonzales et al., 2013) and compounds of plant origin (Khaki et al., 2009; Khaki et al., 2010; Roy et al., 2013) have shown a significant improvement in spermatogenesis, in which sperm count, normal morphology, viability and motility are significantly increased (Tables 1 and 2). Furthermore, curcumin (Kanter et al., 2013) and quercetin (Kanter et al., 2012) have improved spermatogenesis, as shown by an increase in mean testicular biopsy score after 8 weeks of treatment. Several studies have demonstrated the beneficial effects of plantbased products on DM-induced oxidative stress in the sperm and testis of rats. Studies have shown that administration of root extract of Chlorophytum borivilianum for 28 days reduces nitric oxide, hydrogen peroxide and MDA levels in the sperm of STZ induced T1 diabetic rats (Thakur et al., 2009). Khaki et al. (2009) have demonstrated that quercetin decreases MDA concentration and increases total antioxidant capacity of sperm in STZ-induced T1 diabetic rats. Studies using other plant extracts have yielded similar results in the testicular tissue. For example, the combined root extract of Musa paradisiaca and leave extract of Coccinia indica improves antioxidant enzymes activities and reduces lipid peroxidation in the testis of $\mathrm{T} 1$ diabetic rats after 45 days of administration (Mallick et al., 2010).

Testicular germ cell apoptosis is reported to be lower in diabetic rats treated with Musa paradisiaca + Coccinia indica (Mallick et al., 2010) and Eugenia jambolana (Ghosh et al., 2014). Similarly, Thakur et al. (2009) have reported a decrease in apoptosis of spermatozoa of $\mathrm{T} 1$ diabetic rats following 28 days administration of Chlorophytum borivilianum root extract. In addition, the activity of caspase-3 in the spermatozoa is significantly decreased compared to the diabetic untreated rats (Thakur et al., 2009). Studies using phenolic compounds have also yielded a similar trend of results. Administration of curcumin (Kanter et al., 2013) or quercetin (Kanter et al., 2012) for 8 weeks decreases apoptotic index in the testis of $\mathrm{T} 1$ diabetic rats. Administration of scutellarin for 60 days down-regulates Bax protein and up-regulates Bcl-2 in $\mathrm{T} 2$ diabetic rats (Long et al., 2015). Administration of naringenin significantly decreases the number of TUNEL-positive cells in the testis (Roy et al., 2013), implying a decrease in testicular germ cell apoptosis.

\section{Natural products with post-testicular beneficial effects in diabetes mellitus}

Natural products have demonstrated significant beneficial effects in treating impaired sexual behaviour associated with DM (Tables 1 and 2). For example, Dracaena arborea root bark and Mucuna pruriens seeds extract significantly improve erectile function in $\mathrm{T} 1$ diabetic rats after 3 weeks and 40 days of treatment, respectively, compared to the DM untreated rats (Suresh and Prakash, 2012; Wankeu-Nya et al., 2014). Similarly, flavonoid rutin increases cGMP concentration in the homogenate of the penile tissue of $\mathrm{T} 1$ diabetic rats after 5 weeks of treatment (Al-Roujeaie et al., 2016). The root extract of Chlorophytum borivilianum decreases mount, intromission and ejaculation 
latencies, and increases their frequencies in T1 diabetic animal models (Thakur et al., 2009; Vyawahare et al., 2009). WankeuNya et al. (2014) have reported significant decreases in mount, intromission and ejaculatory latencies after 4 weeks of treatment of T1 diabetic rats with root bark extract of Dracaena arborea. Curculigo orchioides extract significantly decreases mount, intromission and ejaculatory latencies and frequencies in $\mathrm{T} 1$ diabetes, (Thakur et al., 2012). Similarly, rutin significantly reduces mount, intromission and ejaculatory latencies, and increases their frequencies (Al-Roujeaie et al., 2016). These results may suggest that natural products can ameliorate DMinduced impaired sexual behaviour and erectile dysfunction. Nevertheless, further studies are suggested to be carried out using these natural products to elucidate their mechanism of actions.

\section{NATURAL PRODUCTS AND BLOOD GLUCOSE REGULATION IN DIABETES MELLITUS}

A greater percentage of the natural products examined in this review significantly reduces fasting blood glucose of diabetic rats as compared to their diabetic untreated counterparts (Table 1). This observation is in addition to their significant beneficial effects on male reproductive function. Several studies have shown that natural products cause pancreatic $\beta$-cells regeneration and improve the secretion and viability of insulin (Giribabu et al., 2014; Koneri et al., 2014). Additionally, natural products by virtue of the fact that most of them contain flavonoids and phenolic compounds, have antioxidant property to reduce the oxidative stress associated with DM (Giribabu et al., 2014; Ogunyinka et al., 2016).

\section{CONCLUSION AND FUTURE DIRECTION}

In conclusion, the studies discussed in this review have suggested a beneficial effect of natural products on DM-induced reproductive abnormalities at the pre-testicular, testicular and posttesticular levels. However, it is suggested to also include studies on their phytochemical compounds, toxicity as well as standard anti-diabetic agent(s) as positive controls to better appreciate their potential to attenuate DM-induced impairment in reproductive function.

\section{ACKNOWLEDGEMENT}

Financial support and sponsorship: The authors hereby acknowledge Universiti Sains Malaysia (USM) for the award of USM Global Fellowship to V. U. Nna.

Conflict of Interests: There are no conflicts of interest.

\section{REFERENCES}

Abbasi Z, Tabatabaei SR, Mazaheri Y, Barati F, Morovvati H. Effects of sesame oil on the reproductive parameters of diabetes mellitusinduced male rats. World J Men's Health, 2013; 31:141-149.

Agbaje IM, Rogers DA, McVicar CM, McClure N, Atkinson $\mathrm{AB}$, Mallidis $\mathrm{C}$, Lewis SE. Insulin dependent diabetes mellitus: implications for male reproductive function. Hum Reprod, 2007; 22:18711877.
Ahangarpour A, Oroojan AA, Heidari H, Ehsan G, Nooshabadi R, Reza M. Effects of hydro-alcoholic extract of Rhus coriaria (Sumac) seeds on reproductive complications of nicotinamide-streptozotocin induced type-2 diabetes in male mice. World J Men's Health, 2014; 32:151-158.

Ahangarpour A, Oroojan AA, Heidari H, Ghaedi E, Taherkhani R. Effects of hydro-alcoholic extract from Arctium lappa L.(Burdock) root on gonadotropins, testosterone, and sperm count and viability in male Mice with nicotinamide/streptozotocin-induced type 2 diabetes. Malays. J Med Sci, 2015; 22:25-32.

Al-Roujeaie AS, Abuohashish HM, Ahmed MM, Alkhamees OA. Effect of rutin on diabetic-induced erectile dysfunction: Possible involvement of testicular biomarkers in male rats. Andrologia, 2016; doi: 10.1111/and.12737.

Arikawe AP, Oyerinde A, Olatunji-Bello II, Obika LF. Streptozotocin diabetes and insulin resistance impairment of spermatogenesis in adult rat testis: central vs local mechanism. Nig J Physiol Sci, 2012; 27:171-179.

Baccetti B, La Marca A, Piomboni P, Capitani S, Bruni E, Petraglia F, De Leo V. Insulin-dependent diabetes in men is associated with hypothalamo-pituitary derangement and with impairment in semen quality. Hum Reprod, 2002; 17:2673-2677.

Bener A, Al-Ansari AA, Zirie M, Al-Hamaq AO. Is male fertility associated with type 2 diabetes mellitus?. Int Urol Nephrol, 2009; 41:777-784.

Cameron DF, Rountree J, Schultz RE, Repetta D, Murray FT. Sustained hyperglycemia results in testicular dysfunction and reduced fertility potential in BBWOR diabetic rats. Am J Physiol Endocrinol Metab, 1990; 259:E881-E889.

Chatterjee K, Ali KM, De D, Bera TK, Jana K, Maiti S, Ghosh A, Samanta R, Ghosh D. Diabetes induced testicular dysfunction amelioration by ethyl acetate fraction of hydromethanolic extract of root of Musa paradisiaca L. in streptozotocin-induced diabetic rat. Asian Pac J Trop Dis, 2012; 2:S233-S241.

Cooke DW, Plotnick L. Type 1 diabetes mellitus in pediatrics. Pediatr Rev, 2008; 29:374-384.

Delfino M, Imbrogno N, Elia J, Capogreco F, Mazzilli F. Prevalence of diabetes mellitus in male partners of infertile couples. Italy $\mathrm{J}$ Urol Nephrol, 2007; 59:131-135.

Feng SL, Li SH, Wang Y, Chen CC, Gao B. Effect of Ligustrum fruit extract on reproduction in experimental diabetic rats. Asian J Androl, 2001; 3:71-73.

Garcia-Diez LC, Corrales Hernandez JJ, Hernandez-Diaz J, Pedraz MJ, Miralles JM. Semen characteristics and diabetes mellitus: significance of insulin in male infertility. Arch Androl, 1991; 26:119-28.

Ghlissi Z, Hamden K, Saoudi M, Sahnoun Z, Zeghal KM, El Feki A, Hakim A. Effect of Nigella sativa seeds on reproductive system of male diabetic rats. Afr J Pharm Pharmacol; 2012; 6:1444-1450.

Ghosh A, Jana K, Ali KM, De D, Chatterjee K, Ghosh D. Corrective role of Eugenia jambolana on testicular impairment in streptozotocin-induced diabetic male albino rat: An approach through genomic and proteomic study. Andrologia, 2014; 46:296-307.

Giribabu N, Kumar KE, Rekha SS, Muniandy S, Salleh N. Chlorophytum borivilianum root extract maintains near normal blood glucose, insulin and lipid profile levels and prevents oxidative stress in the pancreas of streptozotocin-induced adult male diabetic rats. Int J Med Sci, $2014 ; 11: 1172-1184$.

Gobbo MG, Costa CF, Silva DG, de Almeida EA, Góes RM. Effect of melatonin intake on oxidative stress biomarkers in male reproductive organs of rats under experimental diabetes. Oxid Med Cell Long. 2015; doi:doi.org/10.1155/2015/614579.

Gonzales GF, Gonzales-Castañeda C, Gasco M. A mixture of extracts from Peruvian plants (black maca and yacon) improves sperm count and reduced glycemia in mice with streptozotocin-induced diabetes. Toxicol Mech Method, 2013; 23:509-518.

Hafez DA. Effect of extracts of ginger roots and cinnamon bark on fertility of male diabetic rats. J Am Sci, 2010; 6:940-947.

Hamden K, Carreau S, Jamoussi K, Ayadi F, Garmazi F, Mezgenni N, Elfeki A. Inhibitory effects of 1 $\alpha, 25$ dihydroxyvitamin D3 
and Ajuga iva extract on oxidative stress, toxicity and hypo-fertility in diabetic rat testes. J Physiol Biochem, 2008; 64:231-239.

Idris MH, Budin SB, Osman M, Mohamed J. Protective role of Hibiscus sabdariffa calyx extract against streptozotocin induced sperm damage in diabetic rats. Excli J, 2012; 11:659-669.

Jiang X, Zhang C, Xin Y, Huang Z, Tan Y, Huang Y, Wang Y, Feng W, Li X, Li W, Qu Y. 2013. Protective effect of FGF21 on type 1 diabetes-induced testicular apoptotic cell death probably via both mitochondrial-and endoplasmic reticulum stress-dependent pathways in the mouse model. Toxicol Lett. 219:65-76.

Kanter M, Aktas C, Erboga M. Protective effects of quercetin against apoptosis and oxidative stress in streptozotocin-induced diabetic rat testis. Food Chem Toxicol, 2012; 50:719-725.

Kanter M, Aktas C, Erboga M. Curcumin attenuates testicular damage, apoptotic germ cell death, and oxidative stress in streptozotocin -induced diabetic rats. Mol Nutr Food Res, 2013; 57:1578-1585.

Khaki A, Fathiazad F, Nouri M, Khaki A, Maleki NA, Khamnei HJ, Ahmadi P. Beneficial effects of quercetin on sperm parameters in streptozotocin-induced diabetic male rats. Phytother Res, 2010; 24:12851291.

Khaki A, Khaki AA, Hajhosseini L, Golzar FS, Ainehchi N. The anti-oxidant effects of ginger and cinnamon on spermatogenesis dysfunction of diabetes rats. Afr J Trad Comp Alt Med, 2014; 11:1-8.

Khaki AR, Nouri M, Fathiazad F, Ahmadi-Ashtiani HR, Rastgar HO, Rezazadeh S. Protective effects of quercetin on spermatogenesis in streptozotocin-induced diabetic rat. J Med Plants, 2009; 1:57-64.

King H. First International Forum for Diabetes Outcome Research; 1999 Apr 22-23. Copenhagen: Denmark International Diabetes Monitor, 1999.

Koneri RB, Samaddar S, Ramaiah CT. Antidiabetic activity of a triterpenoid saponin isolated from Momordica cymbalaria Fenzl. Indian J Exp Biol, 2014; 52:46-52.

Kumar V, Fausto N, Abbas AK, Cotran RS, Robbins SL. Robbins and Cotran Pathologic Basis of Disease (7th ed.). Philadelphia, Pa.: Saunders, 2005; 1194-1195.

Kyathanahalli CN, Manjunath MJ. Oral supplementation of standardized extract of Withania somnifera protects against diabetesinduced testicular oxidative impairments in prepubertal rats. Protoplasma, 2014; 251:1021-1029.

La Vignera S, Di Mauro M, Condorelli R, La Rosa S, Vicari E. Diabetes worsens spermatic oxidative stress associated with the inflammation of male accessory sex glands. La Clinica Terapeutica, 2008; 160:363-366.

Long L, Wang J, Lu X, Xu Y, Zheng S, Luo C, Li Y. Protective effects of scutellarin on type II Diabetes Mellitus-induced testicular damages related to reactive oxygen species/Bcl-2/Bax and reactive oxygen species/microcirculation/staving pathway in diabetic rat. J Diab Res, 2015; doi: $10.1155 / 2015 / 252530$.

Mallick C, Bera TK, Ali KM, Chatterjee K, Ghosh D. Diabetesinduced testicular disorders vis-a-vis germ cell apoptosis in albino rat: Remedial effect of hexane fraction of root of Musa paradisiaca and leaf of Coccinia indica. J Health Sci, 2010; 56:641-654.

Mallidis C, Agbaje I, McClure N, Kliesch S. The influence of diabetes mellitus on male reproductive function: a poorly investigated aspect of male infertility. Der Urologe Ausg. A, 2011; 50: 33-37.

Mohamed M, Sulaiman SA, Jaafar H, Sirajudeen KN. Antioxidant protective effect of honey in cigarette smoke-induced testicular damage in rats. Int J Mol Sci, 2011; 2:5508-5521.

Mohamed M, Sulaiman SA, Jaafar H. Histological changes in male accessory reproductive organs in rats exposed to cigarette smoke and the protective effect of honey supplementation. Afr J Trad Comp Alt Med, 2012; 9:329-335

Mohamed M, Sulaiman SA, Sirajudeen KN. Protective effect of honey against cigarette smoke induced-impaired sexual behavior and fertility of male rats. Toxicol Ind Health, 2013; 29:264-271.

Mohasseb M, Ebied S, Yehia MA, Hussein N. Testicular oxidative damage and role of combined antioxidant supplementation in experimental diabetic rats. J Physiol Biochem, 2011; 67:185-194.
Nah WH, Koh IK, Ahn HS, Kim MJ, Kang HG, Jun JH, Gye MC. Effect of Spirulina maxima on spermatogenesis and steroidogenesis in streptozotocin-induced type I diabetic male rats. Food Chem, 2012; 134:173-179.

National Health and Morbidity Survey. http://www.iku.gov.my/index.php/research-eng/list-of-research-eng/ikueng/nhms-eng/nhms-2015 (assessed on 16 March 2016), 2015.

Ogunyinka BI, Oyinloye BE, Osunsanmi FO, Opoku AR, Kappo AP. Modulatory influence of Parkia biglobosa protein isolate on testosterone and biomarkers of oxidative stress in brain and testes of streptozotocin-induced diabetic male rats. Int $\mathrm{J}$ Physiol Pathophysiol Pharmacol, 2016; 8:78-86.

Oliveira PF, Alves MG, Rato L, Laurentino S, Silva J, Sa R, Barros A, Sousa M, Carvalho RA, Cavaco JE, Socorro S. Effect of insulin deprivation on metabolism and metabolism-associated gene transcript levels of in vitro cultured human Sertoli cells. Biochim Biophys Acta, 2012; 1820:84-89.

Pitteloud N, Hardin M, Dwyer AA, Valassi E, Yialamas M, Elahi D, Hayes FJ. Increasing insulin resistance is associated with a decrease in Leydig cell testosterone secretion in men. J Clin Endocrinol Metab, 2005; 90:2636-2641.

Rato L, Alves MG, Duarte AI, Santos MS, Moreira PI, Cavaco JE, Oliveira PF. Testosterone deficiency induced by progressive stages of diabetes mellitus impairs glucose metabolism and favors glycogenesis in mature rat Sertoli cells. Int J Biochem Cell Biol, 2015; 66:1-10.

Reddy KP, Rao MN, Murthy JS, Reddy PS. Lead aggravates the diabetic-induced reproductive toxicity in male Wistar rats. Toxicol Res, 2016; 5:1465-1476

Riera MF, Galardo MN, Pellizzari EH, Meroni SB, Cigorraga SB. Molecular mechanisms involved in Sertoli cell adaptation to glucose deprivation. Am J Physiol Endocrinol Metab, 2009; 297:E907-E914.

Roy S, Rahaman N, Ahmed F, Metya S, Sannigrahi S. Naringenin attenuates testicular damage, germ cell death and oxidative stress in streptozotocin induced diabetic rats: naringenin prevents diabetic rat testicular damage. J Applied Biomed, 2013; 11:195-208.

Sangameswaran B, Jayakar B. Anti-diabetic, antihyperlipidemic and spermatogenic effects of Amaranthus spinosus Linn. on streptozotocin-induced diabetic rats. J Nat Med, 2008; 62:79-82.

Saumya SM, Basha PM. Fluoride exposure aggravates the testicular damage and sperm quality in diabetic mice: protective role of ginseng and banaba. Biol Trace Elem Res, 2016; doi: 10.1007/s12011016-0893-y

Shahreari S, Khaki A, Ahmadi-Ashtiani HR, Rezazadeh S, Hajiaghaei R. Effects of Danae racemosa on testosterone hormone in experimental diabetic rats. J Med Plants, 2010; 3:114-119.

Shalaby MA, Hamowieh AR. Safety and efficacy of Zingiber officinale roots on fertility of male diabetic rats. Food Chem Toxicol, 2010; 48:2920-2924.

Srivastava SP, Pant N. Testicular and spermatotoxic effects of qunalphos in rats. J Applied Toxicol, 2003; 23:271-274.

Suresh S, Prakash S. Effect of Mucuna pruriens (Linn.) on sexual behaviour and sperm parameters in streptozotocin-induced diabetic male rat. J Sex Med, 2012; 9:3066-3078.

Thakur M, Bhargava S, Praznik W, Loeppert R, Dixit VK. Effect of Chlorophytum borivilianum Santapau and Fernandes on sexual dysfunction in hyperglycemic male rats. Chinese J Int Med, 2009; 15:448453.

Thakur M, Chauhan NS, Sharma V, Dixit VK, Bhargava S. Effect of Curculigo orchioides on hyperglycemia-induced oligospermia and sexual dysfunction in male rats. Int J Impot Res, 2012; 24:31-37.

Vignon F, Le Faou A, Montagnon D, Pradignac A, Cranz C, Winiszewsky P, Pinget M. Comparative study of semen in diabetic and healthy men. Diab Metab, 1990; 17:350-354.

Vyawahare NS, Kagathara VG, Kshirsagar AD, Rajendran R, Patil MN, Jagtap AA, Sadar SS. Effect of hydroalcoholic extract of Chlorophytum borivilianum tubers in alleviating the diabetic impotency in streptozotocin induced male diabetic rats. Pharmacog Res, 2009; 1:314319. 
Wankeu-Nya M, Florea A, Bâlici S, Watcho P, Matei H, Kamanyi A. Dracaena arborea alleviates ultra-structural spermatogenic alterations in streptozotocin-induced diabetic rats. BMC Comp Alt Med, 2013; doi: 10.1186/1472-6882-13-71.

Wankeu-Nya M, Watcho P, Nguelefack TB, Carro-Juarez M, Tapondjou L, Kamanyi A. Effects of Dracaena arborea (Dracaenaceae) on sexual dysfunction in 4 weeks hyperglycemic male rats. Asian Pac J Trop Med, 2014; 7:609-619.

Young L, Yu D, Bateman RM, Brock GB. Oxidative stress and antioxidant therapy: Their impact in diabetes-associated erectile dysfunction. J Androl, 2004; 25:830-836.

Yu W, Wan Z, Qiu XF, Chen Y, Dai YT. Resveratrol, an activator of SIRT1, restores erectile function in streptozotocin-induced diabetic rats. Asian J Androl, 2013; 15:646-651.

Zaini A. Where is Malaysia in the midst of the Asian epidemic of diabetes mellitus? Diab Res Clin Prac, 2000; 50:S23-S28.
Zhao Y, Tan Y, Dai J, Li B, Guo L, Cui J, Wang G, Shi X, Zhang X, Mellen N, Li W. Exacerbation of diabetes-induced testicular apoptosis by zinc deficiency is most likely associated with oxidative stress, p38 MAPK activation, and p53 activation in mice. Toxicol Lett, 2011; 200:100-106

Zhu W, Chen M, Shou Q, Li Y, Hu F. Biological activities of Chinese propolis and Brazilian propolis on streptozotocin-induced type 1 diabetes mellitus in rats. Evid-Based Comp Alt Med, 2011; doi: 10.1093/ecam/neq025.

\section{How to cite this article:}

Nna VU, Bakar AB, Mohamed M. Diabetes mellitus-induced male reproductive impairment: The role of natural products (A review). J App Pharm Sci, 2017; 7 (09): 233-242. 